\title{
Hypertension and Future Cardiovascular Health in Pediatric End-Stage Renal Disease Patients
}

\author{
Joseph T. Flynn \\ University of Washington School of Medicine, and Pediatric Hypertension Program, Seattle Children's Hospital, \\ Seattle, Wash., USA
}

\section{Key Words}

Blood pressure $\cdot$ Children $\cdot$ Adolescents $\cdot$ Hypertension •

Cardiovascular disease $\cdot$ Dialysis

\begin{abstract}
Background: There are now numerous studies that have documented an increased risk of cardiovascular morbidity and mortality in young adults who had childhood-onset end-stage renal disease (ESRD). Since the number of such patients surviving to adulthood is increasing, strategies to reduce this risk are urgently needed. Methods: The various risk factors contributing to adult cardiovascular disease in this population will be reviewed, with an emphasis on hypertension and its control. Data demonstrating the prevalence of hypertension in childhood chronic kidney disease as well as the results of improved blood pressure control in ESRD will also be presented. Conclusions: Hypertension is exceedingly common in pediatric ESRD patients and frequently poorly controlled. Efforts to improve blood pressure control in this patient population could potentially reduce future cardiovascular morbidity and mortality.
\end{abstract}

Copyright $\odot 2012$ S. Karger AG, Base
(๑) 2012 S. Karger AG, Basel 0253-5068/12/0333-0138\$38.00/0

Fax +4161306 1234 E-Mail karger@karger.ch www.karger.com

\section{Pediatric Origins of Increased Cardiovascular Risk}

It is well known that mortality rates among adults with end-stage renal disease (ESRD), particularly those receiving dialysis, are significantly greater than among agematched controls in the general population. Cardiovascular causes and infections remain the most common causes of death in these patients, although recent data have shown a slight decrease in the rates of cardiovascular-related hospitalizations and death [1].

What is less well known, particularly outside of the pediatric nephrology community, is that for at least some adults with ESRD, the increase in cardiovascular morbidity and mortality may actually begin in childhood. Specifically, cardiovascular disease has been established as the leading cause of death in adults with childhood-onset ESRD. Initial data demonstrating this phenomenon came from analysis of data from the United States Renal Data Systems, which collects information on essentially all US ESRD patients. In this study [2], among 1,380 patients with childhood-onset ESRD who died before 30 years of age, $23 \%$ of deaths were cardiovascular in origin: the cardiovascular death rate was 1,000 times higher among children with ESRD than in the general population, and

Prof. Joseph T. Flynn, MD, MS

Division of Nephrology, Seattle Children's Hospital

4800 Sand Point Way NE

Seattle, WA 98105 (USA)

Tel. +1 206987 2524, E-Mail joseph.flynn@ seattlechildrens.org 
100 times higher among young adults with ESRD than in the general population. The early onset of cardiovascular complications was subsequently demonstrated by Chavers et al. [3], who documented high rates of cardiovascular complications in pediatric dialysis patients beginning in childhood.

Additional data have been provided by the Late Effects of Renal Insufficiency in Children Study, a cohort study of 249 Dutch adult patients with onset of ESRD between 0 and 14 years of age [4]. The overall mortality risk of the ESRD patients was 31 times that of age-matched Dutch citizens, and cardiovascular disease accounted for $41 \%$ of all mortalities, with cardiac death becoming the most common cause of mortality after 10 years of receiving renal replacement therapy [4]. Given the increase in survival of pediatric patients with ESRD to adulthood [5], the number of such adults at increased cardiovascular risk is sure to increase in the coming decades.

While the observational studies cited earlier cannot provide explanations for the astonishingly high rates of cardiovascular disease in children and young adults with ESRD, it is also well established that chronic kidney disease $(\mathrm{CKD})$ is associated with numerous traditional and nontraditional cardiovascular risk factors, even in pediatric patients (table 1). Traditional risk factors that are especially pertinent in pediatric patients with CKD include the following: male gender, as there is a male predominance in pediatric CKD [6]; black race, as evidenced by the previously cited studies $[2,3]$ that showed increased rates of cardiovascular disease in black versus non-black children; hypertension, which will be reviewed in detail below; and dyslipidemia, the prevalence of which is only now beginning to be understood among children with mild-to-moderate CKD [7].

Obesity is also an interesting issue. While many children with CKD experience growth failure, recent data from the Chronic Kidney Disease in Children (CKiD) study show a higher than expected prevalence of overweight [6]. However, whether a significant number of these children exhibit the typical central obesity associated with elevated cardiovascular risk in adults is unknown.

Nontraditional cardiovascular risk factors are also becoming increasingly important in pediatric patients with CKD [8]. Of those listed in table 1, the one that has received the greatest attention is disordered calciumphosphorus metabolism leading to vascular calcification. Treatment of secondary hyperparathyroidism with calcium-containing phosphate binders and vitamin D analogues can lead to hypercalcemia as well as an ele-
Table 1. Traditional and nontraditional cardiovascular risk factors in patients with CKD

\begin{tabular}{ll}
\hline Traditional & Nontraditional \\
\hline Age & Renal insufficiency \\
Race & Microalbuminuria/proteinuria \\
Male gender & Hyperparathyroidism \\
Hypertension & Anemia \\
Dyslipidemia & Impaired glucose metabolism \\
Diabetes mellitus & Chronic inflammation \\
Tobacco use & Hyperhomocysteinemia \\
Sedentary lifestyle & Thrombotic alterations \\
Psychosocial stress & Endothelial dysfunction \\
Family history & Fluid overload \\
Left ventricular hypertrophy & Decreased adiponectin \\
Obesity & Vascular calcification \\
\hline
\end{tabular}

vated calcium-phosphorus product, which in turn can produce extensive soft tissue calcifications, the most alarming of which have been shown to occur in the coronary arteries [9]. Abnormal calcium-phosphorus metabolism was found to be correlated with increased carotid intima media (IMT) thickness and vascular dysfunction in both pre-ESRD and ESRD patients by Mitsnefes et al. [10]. Other nontraditional risk factors, such as inflammation, decreased adiponectin, and impaired glucose tolerance, are also present in pediatric patients with CKD.

It is thought that the combination of these many risk factors, especially over many years of CKD and renal replacement therapy, leads to an acceleration in various pathophysiological processes, in particular atherosclerosis and myocardial hypertrophy. This has been reviewed in detail elsewhere [11]. The task for the clinician is to identify which cardiovascular risk factors are present in an individual patient and institute appropriate changes in management to reduce the patient's overall cardiovascular risk.

\section{Hypertension in Pediatric CKD}

Hypertension is perhaps one of the most common of the traditional cardiovascular risk factors seen in young CKD/ESRD patients, and is a risk factor that should be modifiable. Until recently, however, reliable data on the prevalence of hypertension in children with CKD or ESRD were limited. For pediatric CKD, for example, most available information on blood pressure (BP) came 
from registry reports such as those from the North American Pediatric Renal Trials and Collaborative Studies, which demonstrated substantial rates of hypertension whether hypertension was defined as treatment with antihypertensive medications or as elevated measured BP [12]. More complete data has been provided by the prospective observational CKiD cohort study [13], which demonstrated that approximately $54 \%$ of children with CKD demonstrate hypertension (defined as measured BP $\geq 95$ th percentile and/or history of hypertension with antihypertensive medication use), with many more children demonstrating measured BP $>90$ th percentile, the current recommended goal BP for children with CKD [12].

Data on BP levels in pediatric ESRD patients has also come primarily from registry reports. Mitsnefes et al. [14] reported that about $60 \%$ of pediatric dialysis patients had uncontrolled hypertension, defined as measured BP $\geq 95$ th percentile. Young age, recent dialysis initiation, and hemodialysis (HD) modality were identified as risk factors for having uncontrolled BP. Similar poor control of hypertension in American dialysis patients has been reported by Chavers et al. [15] for HD patients and Halbach et al. [16] for both HD and peritoneal dialysis patients. In the latter study, demographic factors, such as young age and black race, and treatment factors, such as prescription of antihypertensive medications, were also identified as risk factors for poorly controlled hypertension. Both of these studies are notable for their reliance upon registry data; thus, there is limited information available on the technique and/or frequency of BP measurement, or the goals of hypertension management.

Fewer data are available on pediatric transplant recipients; most reports to date come from single-center studies such as that of Sinha et al. [17], who reported a prevalence of hypertension of over $50 \%$ among pediatric renal transplant recipients followed at Great Ormond Street Hospital. In that study, uncontrolled hypertension was more likely, and the number of antihypertensive medications greater, among children with more severe CKD. While this study is notable for the consistency of BP measurement, the study population was small, limiting the conclusions that can be drawn regarding factors affecting BP control.

More recently, Kramer et al. [18] reported data from the ESPN/ERA-EDTA registry on BP control among pediatric ESRD patients in Europe, including patients receiving $\mathrm{HD}$, peritoneal dialysis, and postrenal transplant. They confirmed the poor control of hypertension report- ed in the earlier studies noted above; specifically, hypertension was present in $69.4 \%$ of $\mathrm{HD}, 68.6 \%$ of peritoneal dialysis, and $66.9 \%$ of transplant recipients. Among dialysis patients, their data confirm earlier reports that younger age, recent dialysis initiation, and HD modality are risk factors for poor BP control among the dialysis group; the best control of BP was seen among transplant recipients.

Taken together, the above studies clearly demonstrate that hypertension is common among children with CKD/ ESRD, and that in some patients, it may be undertreated or even unrecognized. Thus, it is proper to focus on hypertension as a potentially modifiable risk factor in this patient population.

\section{Cardiovascular Consequences of Hypertension in Pediatric CKD}

'Hard' cardiovascular consequences of hypertension such as myocardial infarction, stroke, or death are relatively uncommon during childhood, even among patients with long-standing CKD. In the report by Chavers et al. [3], the most common cardiovascular 'events' were arrhythmias, valvular disease, and cardiomyopathy; cardiac deaths accounted for just $9 \%$ of all reported events. Thus, most pediatric studies have focused on intermediate outcomes of hypertension such as left ventricular hypertrophy (LVH) and atherosclerosis.

One of the first studies to demonstrate that a significant percentage of children and young adults with CKD have LVH and/or other signs of cardiomyopathy was that of Johnstone et al. [19]. They found that patients with CKD had significantly increased left ventricular mass index (LVMI) compared to normal controls, and that creatinine appeared to correlate with LVMI. Even greater increases in LVMI were seen in dialysis patients, and abnormal LVMI persisted even after renal transplantation. However, no correlation was seen between BP and increased LVMI in that study. These findings have been duplicated and extended by Mitsnefes et al. [20-22], who in a series of single-center studies demonstrated increased left ventricular mass in pediatric predialysis CKD [20] in patients on dialysis [21] or after renal transplantation [22]. While different associations with several of the cardiovascular risk factors listed in table 1 were noted in these reports, BP was consistently found to be a significant predictor of increased LVMI.

The significance of hypertension in the development of $\mathrm{LVH}$ in pediatric CKD is underscored by two other re- 
cent studies of children with moderate CKD. In a singlecenter study of 49 patients with a mean estimated GFR of $26 \mathrm{ml} / \mathrm{min} / 1.73 \mathrm{~m}^{2}$, Sinha et al. [23] demonstrated that higher BP, even if not in the overtly hypertensive range, was strongly associated with the development of LVH. The CKiD investigators reported their findings in 198 children studied by both echocardiography and ambulatory BP monitoring (ABPM) [24]. They showed a higher prevalence of LVH in children with either masked or confirmed ambulatory hypertension, both of which were more closely associated with LVH than office BP readings.

Evidence linking hypertension and the development of atherosclerosis in pediatric CKD/ESRD has been lacking to date. Several studies have demonstrated either overt atherosclerosis by histologic examination of vessels [25], or indirect evidence in the form of increased vessel IMT [26]; however, nontraditional risk factors have been more strongly correlated with the vascular abnormalities in these studies than BP. One study of children with CKD, some of whom were predialysis, some on dialysis, and some post-transplant, showed that BP correlated with carotid IMT, but only in the predialysis and dialysis groups (fig. 1) [27]. These investigators also noted strong correlations between serum phosphate and carotid IMT, confirming the strong relationship between nontraditional risk factors and atherosclerosis mentioned previously. Clearly further studies, probably longitudinal in nature, will be needed to better understand the relationship between BP and the development of atherosclerosis in this patient population.

\section{How Can Future Cardiovascular Risk Be Reduced?}

Given the many potential cardiovascular risk factors that may be present in a patient with CKD/ESRD, a multifactorial individualized approach to risk reduction will be needed. This may include advice on lifestyle changes, such as smoking cessation and weight loss, and prescription of various medications, including antihypertensives, phosphate binders, and lipid-lowering agents. The interested reader is referred to recent comprehensive reviews for more information $[11,28]$.

With respect to hypertension specifically, few data are available on whether sustained control of hypertension can be achieved in pediatric CKD and ESRD patients, or whether such control will have long-term benefits. Ideally, a large cohort of patients such as that enrolled in the CKiD study should be followed over the course of their

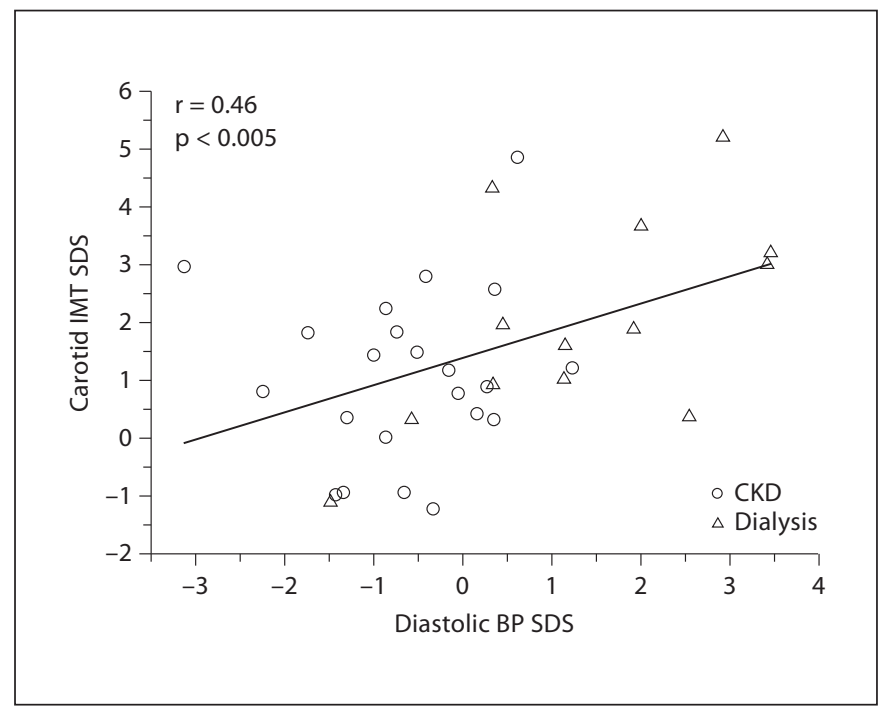

Fig. 1. Relationship between diastolic BP and carotid IMT in pediatric patients with CKD or on dialysis. Reprinted from Litwin et al. [27] with permission from Oxford University Press.

CKD and progression through dialysis and transplant, with follow-up extended into adulthood. To date, no such study has been conducted. However, interesting single-center data have recently been published by a group at the Karolinska Institute [29] that indicate longterm cardiovascular risk reduction may indeed be possible in patients with childhood-onset CKD. They followed 22 renal transplant recipients over 9 years with sequential carotid ultrasounds and echocardiograms; hypertension treatment was guided by annual ABPM. With this protocol, they were able to achieve a remarkably low prevalence of LVH of just $4.5 \%$, and saw no change in carotid IMT over the study period. While a prospective, multicenter study should ideally be conducted to confirm these findings, it does appear that long-term reduction of cardiovascular risk can be achieved with appropriate patient monitoring and strict control of BP.

In the meantime, how should the problem of poor $\mathrm{BP}$ control among pediatric CKD and ESRD patients be addressed by the clinician so that future cardiovascular morbidity and mortality can be reduced? The available literature suggests several approaches: first, BP in this population needs to be carefully and consistently measured so that reliable BP data will be available to guide treatment. Auscultation remains the gold standard method of BP measurement in pediatrics and can be successfully utilized in both clinical and research set- 
tings [13]. ABPM adds important additional information to office BP measurement, especially in patients with CKD $[24,30]$. It is our recommendation that ABPM be performed in all patients with mild-moderate CKD in order to most accurately detect elevated BP, and we recommend that repeat $\mathrm{ABPM}$ be utilized to guide therapy.

Second, greater efforts should be made to control BP among young CKD and ESRD patients, particularly within the first year of starting renal replacement therapy. It appears from several studies $[16,18]$ that younger patients with CKD/ESRD have more poorly controlled $\mathrm{BP}$ than older patients, perhaps because of the difficulty of measuring BP and recognizing hypertension in young children. Early control of hypertension may be particularly important in reducing future cardiovascular risk.
Finally, attention should be paid to the class of antihypertensive medications used and the $\mathrm{BP}$ goals chosen. $\mathrm{Pa}$ tients in the CKiD study who were treated with ACE inhibitors or angiotensin receptor blockers had better control of BP compared to patients who did not receive these classes of agents [13]. As has been reviewed elsewhere [12], this is likely due to the central role played by the reninangiotensin-aldosterone system in the pathophysiology of hypertension in patients with CKD. Although there is some controversy regarding the proper BP targets for adults with CKD, pediatric studies have shown that lower BP targets are associated with lower rates of development of LVH [23] and slower rates of CKD progression [12]. Given this, lower BP targets, perhaps between the 50th and 75th percentiles for age, should probably be adopted in young CKD/ESRD patients.

\section{References}

1 US Renal Data System: USRDS 2010 Annual Data Report: Atlas of Chronic Kidney Disease and End-Stage Renal Disease in the United States. Bethesda, National Institutes of Health, National Institute of Diabetes and Digestive and Kidney Diseases, 2010.

-2 Parekh RS, Carroll CE, Wolfe RA, et al: Cardiovascular mortality in children and young adults with end-stage kidney disease. J Pediatr 2002;141:191-197.

-3 Chavers BM, Li S, Collins AJ, Herzog CA: Cardiovascular disease in pediatric chronic dialysis patients. Kidney Int 2002;62:648653.

-4 Groothoff J, Gruppen M, de Groot E, et al: Cardiovascular disease as a late complication of end-stage renal disease in children. Perit Dial Int 2005;25(suppl 3):S123-S126.

- 5 Kramer A, Stel VS, Tizard J, Verrina E, Rönnholm K, Pálsson R, Maxwell H, Jager KJ: Characteristics and survival of young adults who started renal replacement therapy during childhood. Nephrol Dial Transplant 2009;24:926-933.

-6 Furth SL, Abraham AG, Jerry-Fluker J, Schwartz GJ, Benfield M, Kaskel F, Wong C, Mak RH, Moxey-Mims M, Warady BA: Metabolic abnormalities, cardiovascular disease risk factors, and GFR decline in children with chronic kidney disease. Clin J Am Soc Nephrol 2011;6:2132-2140.

7 Saland JM, Pierce CB, Mitsnefes MM, Flynn JT, Goebel J, Kupferman JC, Warady BA, Furth SL, CKiD Investigators: Dyslipidemia in children with chronic kidney disease. Kidney Int 2010;78:1154-1163.

8 Flynn JT: Cardiovascular disease in children with chronic renal failure. Growth Horm IGF Res 2006;16(suppl A):S84-S90.
-9 Goodman WG, Goldin J, Kuizon BD, Yoon C, Gales B, Sider D, Wang Y, Chung J, Emerick A, Greaser L, Elashoff RM, Salusky IB: Coronary-artery calcification in young adults with end-stage renal disease who are undergoing dialysis. N Engl J Med 2000;342: 1478-1483.

10 Mitsnefes MM, Kimball TR, Kartal J, Witt SA, Glascock BJ, Khoury PR, Daniels SR: Cardiac and vascular adaptation in pediatric patients with chronic kidney disease: role of calcium-phosphorus metabolism. J Am Soc Nephrol 2005; 16:2796-2803.

$\checkmark 11$ Lillen MR, Groothoff JW: Cardiovascular disease in children with CKD or ESRD. Nat Rev Nephrol 2009;5:229-235.

12 Shatat IF, Flynn JT: Hypertension in children with chronic kidney disease. Adv Chron Kid Dis 2005; 12:378-384.

13 Flynn JT, Mitsnefes M, Pierce C, et al: Blood pressure in children with chronic kidney disease: a report from the Chronic Kidney Disease in Children study. Hypertension 2008;52:631-637.

14 Mitsnefes M, Stablein D: Hypertension in pediatric patients on long-term dialysis: a report of the North American Pediatric Renal Transplant Cooperative Study (NAPRTCS). Am J Kidney Dis 2005;45:309-315.

$\checkmark 15$ Chavers BM, Solid CA, Daniels FX, et al: Hypertension in pediatric long-term hemodialysis patients in the United States. Clin J Am Soc Nephrol 2009;4:1363-1369.

16 Halbach S, Martz K, Matoo T, Flynn J: Control of hypertension in pediatric dialysis patients (abstract). J Pediatr 2011; DOI:10.1016/ j.jpeds.2011.09.046.
17 Sinha R, Saad A, Marks SD: Prevalence and complications of chronic kidney disease in paediatric renal transplantation: a K/DOQI perspective. Nephrol Dial Transplant 2010; 25:1313-1320.

18 Kramer AM, van Stralen KJ, Jager KJ, Schaefer F, Verrina E, Seeman T, Lewis MA, Boehm M, Simonetti GD, Novljan G, Groothoff JW: Blood pressure and hypertension in children on renal replacement therapy in Europe. Kidney Int 2011, E-pub ahead of print; DOI: $10.1038 / \mathrm{ki} .2011 .232$.

-19 Johnstone LM, Jones CL, Grigg LE, Wilkinson JL, Walker RG, Powell HR: Left ventricular abnormalities in children, adolescents and young adults with renal disease. Kidney Int 1996;50:998-1006.

20 Mitsnefes MM, Kimball TR, Kartal J, Witt SA, Glascock BJ, Khoury PR, Daniels SR: Progression of left ventricular hypertrophy in children with early chronic kidney disease: 2-year follow-up study. J Pediatr 2006; 149:671-675.

21 Mitsnefes MM, Daniels SR, Schwartz SM, Meyer RA, Khoury P, Strife CF: Severe left ventricular hypertrophy in pediatric dialysis: prevalence and predictors. Pediatr Nephrol 2000;14:898-902.

-22 Mitsnefes MM, Schwartz SM, Daniels SR, Kimball TR, Khoury P, Strife CF: Changes in left ventricular mass index in children and adolescents after renal transplantation. Pediatr Transplant 2001;5:279-284.

23 Sinha MD, Tibby SM, Rasmussen P, Rawlins D, Turner C, Dalton RN, Reid CJ, Rigden SP, Booth CJ, Simpson JM: Blood pressure control and left ventricular mass in children with chronic kidney disease. Clin J Am Soc Nephrol 2011;6:543-551. 
24 Mitsnefes M, Flynn J, Cohn S, Samuels J, Blydt-Hansen T, Saland J, Kimball T, Furth S, Warady B, CKiD Study Group: Masked hypertension associates with left ventricular hypertrophy in children with CKD. J Am Soc Nephrol 2010;21:137-144.

-25 Nayir A, Bilge I, Kilicaslan I, Ander H, Emre $\mathrm{S}$, Sirin A: Arterial changes in paediatric haemodialysis patients undergoing renal transplantation. Nephrol Dial Transplant 2001;16: 2041-2047.
26 Litwin M, Wühl E, Jourdan C, Trelewicz J, Niemirska A, Fahr K, Jobs K, Grenda R, Wawer ZT, Rajszys P, Tröger J, Mehls O, Schaefer F: Altered morphologic properties of large arteries in children with chronic renal failure and after renal transplantation. J Am Soc Nephrol 2005; 16:1494-1500.

27 Litwin M, Wühl E, Jourdan C, Niemirska A, Schenk JP, Jobs K, Grenda R, Wawer ZT, Rajszys P, Mehls O, Schaefer F: Evolution of large-vessel arteriopathy in paediatric patients with chronic kidney disease. Nephrol Dial Transplant 2008;23:2552-2557.

28 Parekh RS, Gidding SS: Cardiovascular complications in pediatric end-stage renal disease. Pediatr Nephrol 2005;20:125-131.
29 Balzano R, Lindblad YT, Vavilis G, Jogestrand T, Berg UB, Krma RT: Use of annual ABPM, and repeated carotid scan and echocardiography to monitor cardiovascular health over 9-years in pediatric and young adult renal transplant recipients. Pediatr Transplant 2011;15:635-641.

30 Dionne JM, Turik MM, Hurley RM: Blood pressure abnormalities in children with chronic kidney disease. Blood Press Monit 2008;13:205-209. 